\title{
New concept of very high energy cosmic ray observation wide field-of-view telescope
}

\author{
Kenji Shinozaki*1, Andii Neronov ${ }^{2}$, Andrea Santangelo ${ }^{1}$ and Simona Toscano ${ }^{2 \dagger}$ \\ ${ }^{1}$ Instiute for Astronomy and Astrophysics, University of Tübingen, Sand 1, D-72076, Germany \\ ${ }^{2}$ ISDC Data Centre for Astrophysics, University of Geneva, Chemin d'Ecogia 16, CH-1290 \\ Versoix, Switzerland \\ E-mail: shinozakilastro.uni-tuebingen.de
}

\begin{abstract}
The chemical composition of the very high energy cosmic rays (VHECRs) is an important piece of information to investigate their origin and acceleration mechanism. Possible change of chemical composition around the knee energy at $\sim$ a few times of $10^{15} \mathrm{eV}$ has been reported by air shower (AS) experiments. So far low flux of VHECRs along with uncertainties due to indirect detection, has limited the precision of chemical composition measurement. It is thus essential to improve the primary mass estimation via the measurement of additional parameters in AS observations. In the present work, we propose a new concept of VHECR observation using ultra-wide fieldof-view (FOV) refractive imaging atmospheric Cherenkov telescopes (IACTs). In the framework of the JEM-EUSO mission, wide FOV optics have been designed for ultra-high energy cosmic ray (UHECR) observation from Space. The pathfinder experiments testing such optics have been successfully operated on balloon and on the ground. Above $\sim 10^{15} \mathrm{eV}$, the Cherenkov light from VHECR initiated ASs spreads over a few $\mathrm{km}$, and it can be detected even by meter-scale telescopes. Cherenkov images of distant ASs may span $\sim 20^{\circ}$ or more and thus wide FOV optics is necessary for their detection and characterization for chemical composition studies. In addition, we also discuss prospective advantages of EUSO-type wide-FOV telescopes for VHECR and UHECR physics.
\end{abstract}

The 34th International Cosmic Ray Conference,

30 July- 6 August, 2015

The Hague, The Netherlands

\footnotetext{
*Speaker.

${ }^{\dagger}$ Now at Vrije Universiteit, Brussels, Belgium.
} 


\section{Introduction}

The origin and acceleration mechanisms of cosmic rays (CRs) are long standing problems in contemporary astrophysics. Energy spectrum, arrival direction distribution and chemical composition of CRs are key types of the data provided by CR experiments. They are used together with gamma ray and neutrino experiments to investigate these problems, in particular aim at search for possible CR sources. CRs with energies below $\sim 10^{14} \mathrm{eV}$ can be directly detected by space- and balloon- borne detectors, allowing to directly identify the particle type of each CR and to distinguish different contributions in the overall flux. At higher energies, the CR flux is too low to be directly measured by particle detectors. Instead, CRs are detected indirectly via the signals from air showers (ASs). In this case measurements of the primary CR energy and particle type have to be derived from the information contained in the secondary AS particles reaching the ground level. They are sampled by surface detector arrays. In addition, Cherenkov and/or fluorescence light from ASs are detected to image AS development by optical means.

These measurements are usually interpreted by comparing with numerical simulations of AS phenomena. Simulations of ASs induced by protons and heavier nuclei are based on the phenomenological models of hadronic interactions that contain uncertainties. While measurements of a larger number of ASs help understand the average properties in the overall CR flux, the observable characteristics of the AS induced by same primary particles largely fluctuate. Thus, ASs induced by different energies or/and primary particles possibly result in similar observed characteristics, introducing uncertainties.

So far, the chemical composition of very high energy cosmic rays (VHECRs) in the so-called "knee" energies around $10^{15}-10^{16} \mathrm{eV}$ and higher, has been mainly measured via comparison of the number of electrons $N_{\mathrm{e}}$ in ASs and that of muons at the ground level. ASs induced by heavier nuclei have more muon content than those by proton. This technique was used by KASCADE ans extended to higher energies by KASCADE-Grande experiment. They reported the changes of the chemical composition of the CRs in this energy range [1] and established existences of knee for protons and light nuclei at $\simeq 3 \times 10^{15} \mathrm{eV}$ [2] and that for heavier component at $\simeq 8 \times 10^{16} \mathrm{eV}$ [3], respectively. The result in Reference [2], supported by Tibet AS- $\gamma$ Collaboration [4], has been later challenged by the ARGO-YBJ collaboration, which observed it at even lower energy [5].

Despite significant uncertainties in these results, mainly stemming from the hadronic interaction models, suggestions on the scaling of the knee energy by the electric charge of primary CRs have deep implications for the nature of the knee. An independent verification of the changes of $\mathrm{CR}$ composition over knee energies and more precise measurements of knee features for individual components are needed for further theoretical understanding.

In the present work, we propose a qualitatively new approach for VHECR observation based on Cherenkov imaging technique. This technique has been successfully used in gamma ray astronomy at $\mathrm{GeV}-\mathrm{TeV}$ energies with imaging atmospheric Cherenkov telescopes (IACTs) such as H.E.S.S., MAGIC and VERITAS [6]. These telescopes are characterized by reflective optics with a few-100$\mathrm{m}^{2}$-scale large reflector. On the hand, FOVs are relatively narrow as $\lesssim 10^{\circ}$.

For VHECR observation, the optics aperture may be as small as a few $\mathrm{m}^{2}$, while the wide FOV is critical to measure AS images that are more elongated than those from gamma ray induced AS. Such telescopes have been designed in the JEM-EUSO mission [7], a proposed pioneering space 
mission devoted to the investigation of ultra-high energy cosmic rays (UHECRs) at $\sim 10^{20} \mathrm{eV}$. The JEM-EUSO telescope consists of three $\sim 5-\mathrm{m}^{2}$ Fresnel lenses, covering $\sim 60^{\circ} \phi \mathrm{FOV}$. It will detect the UV fluorescence light from UHECR induced ASs penetrating in the atmosphere and reflected Cherenkov light. Pathfinder telescopes for the mission have been fabricated with two $\sim 1-\mathrm{m}^{2}$ lenses and are successfully operated in EUSO-Balloon [8] and EUSO-TA [9] experiments. Equipped with an array of multi-anode photomultiplier tubes on the focus, $\sim 11^{\circ} \mathrm{FOV}$ has been achieved.

In the present work, we introduce a new observation scheme for VHECR physics. Proposed experiments for this purpose are based on EUSO-type wide-FOV telescopes used as IACTs. From pilot simulations we demonstrate the feasibility of this technique to observe and study VHECRs.

\section{Cherenkov light images of air showers from very high energy cosmic rays}

High energy particles constituting the AS generate fluorescence and Cherenkov light in the atmosphere. The former is isotropically emitted along the track of charged particle with a characteristic line spectrum in 300-430 $\mathrm{nm}$. As its light intensity decreases with distance by inverse-square law, AS measurements via fluorescence light are only effective to UHECR observation. The latter has a continuum spectrum with $\propto 1 / \lambda^{2}$ and is emitted in the direction close to the AS axis. These photons form a cone with a typical opening angle, or the so-called 'Cherenkov angle', $\theta_{\check{\mathrm{C}}} \sim 1^{\circ}$.

The intensity of Cherenkov emission nearly constant inside the so-called 'Cherenkov light pool' and is far more intense than that of fluorescence emission. The radius of the light pool is approximated by $\sim L_{\mathrm{emit}} \cdot \theta_{\check{\mathrm{C}}}$, where $L_{\mathrm{emit}}$ is a typical distance of the Cherenkov emission point from the observation level. For IACTs operated at sea level, $L_{\mathrm{emit}}$ is typically $\sim 10 \mathrm{~km}$ in the case of sub-TeV gamma ray induced ASs from zenith. Thus, it translates to the radius of $\sim 150 \mathrm{~m}$. Outside the light pool, only weaker Cherenkov signals reach the telescope, making gamma ray observations highly ineffective. This fact allows telescopes with FOV as narrow as several degrees for effective gamma ray observations.

Figure 1 shows typical Cherenkov light images of proton- (top panels) and iron- induced ASs (bottom). Left and right columns denote different core distances, perpendicular distance from the telescope to the AS axis, of $300 \mathrm{~m}$ and $600 \mathrm{~m}$ from the telescope, respectively. The pixel size is set to $0.2^{\circ}$ that is compatible with ones of the EUSO pathfinder telescopes. The origin is the arrival direction of the AS and is shown by the cross. The horizontal line passing this point is the projection of the so-called shower-detector plane (SDP).

ASs induced by hadronic particles show different properties from those from gamma rays. Moreover, longitudinal profiles vary systematically depending on energy and mass number of primary particle. This is well characterized by the atmospheric depth of the AS maximum $X_{\max }$. It grows with the increase of primary energy. In comparison to TeV energies, ASs from VHECRs naturally develops lower altitudes by several kilometers.

Among ASs from different primary particles, $X_{\max }$ decreases with $A$ of the primary particle. In particular, AS induced by a primary CR with mass $A$ and energy $E$ approximately is decomposed onto $\sim A$ 'sub-showers' each of which is from a proton with an energy of $E / A$. Its longitudinal profile is similar to the profile of the AS induced by a proton with $1 / A$ times lower energy, resulting in smaller $X_{\max }$. Following this property, there are qualitative differences between proton and iron induced ASs at the same energy as seen in the figure. Measurements of the $X_{\max }$ distribution 

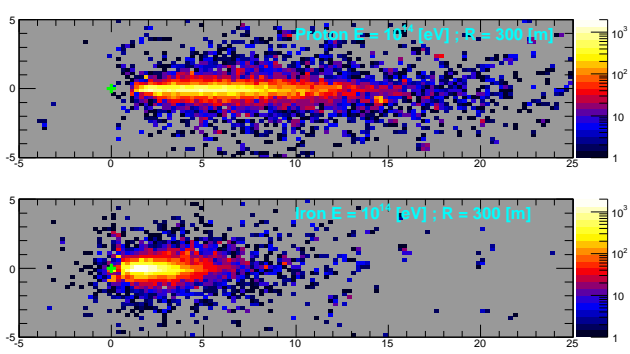
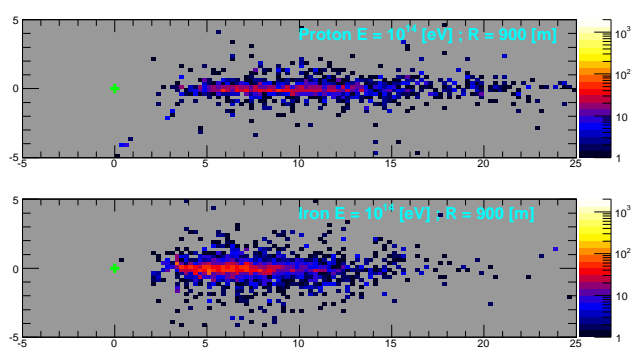

Figure 1: Example of Cherenkov light images of proton (top panels) and iron (bottom) induced ASs hitting the ground at distances of $300 \mathrm{~m}$ (left) and $600 \mathrm{~m}$ (right) from the telescope. The primary energy and arrival direction is set to $10^{14} \mathrm{eV}$ and from zenith, respectively. The pixel size is $0.2^{\circ}$.

provide more direct information on the chemical composition of the overall CR flux. In a similar way, shower-to-shower fluctuations of $X_{\max }$ are smaller for the heavier primaries. These effects are used by the UHECR experiments to estimate the average chemical composition [10].

In hadronic cascade such as a VHECR induced AS, a bunch lower energy particles are produced in each generation of interactions. This leads fast dispersion of the primary energy to the secondary particles than the case of electromagnetic cascade like a gamma ray induced AS. Consequently, the average energy of AS particles, predominantly electrons, decrease much faster. In the atmosphere, trajectories of these electrons are thus more strongly affected by multiple scattering, extending the angular distribution of their trajectory from the AS axis.

In general, ASs from heavier nucleus have wider spreads of secondary particles in comparison with those from proton. Thus, the lateral distribution and its evolution along AS development serve another important information on primary particles. At the energies of interest, the Cherenkov light pool is not clearly formed in ASs from VHECRs. As a result, the emitted Cherenkov light may reach even further distances than the case of gamma ray induced ASs. These factors significantly enhance the instantaneous aperture for AS detection by each telescope.

\section{Analysis schemes}

The analysis design discussed in the present work is based on the detection of the Cherenkov light signals from the observed AS, while in reality there are contamination of noises from the night sky background and other additional sources. These contributions and the fluorescence emission from AS have not been taken into account since the scope of the present work to demonstrate the key concepts of the new observation technique. Pilot simulations have been made using CORSIKA code [11] complied with the Cherenkov standard option [12] along with hadronic interaction models of QGSJET II-04 [13] and FLUKA [14]. We also assume that the geometry of the AS is known by stereoscopic observation, using an array of multiple IACTs or/and by other hybrid method based on the surface detector array data. Further investigations to optimize the detector configurations, alignment of telescopes are in progress. Under these assumptions, we introduce two data analysis sachems, i.e. the 'Hillas-type analysis' and the 'forward Nerling approach'.

Hillas-type analysis The analysis characterizes the shape of the image formed in the camera on the focus using the 'Hillas parameters' [15]. It is a common and well established analysis method 
in ground-based gamma ray astronomy. For $s_{i}$ being the signals, i.e. the number of photons or photoelectrons, detected in $i$-th pixel, SIZE is defined by the sum of all signals as follows:

$$
(\mathrm{SIZE})=\sum^{\mathrm{FOV}} s_{i}
$$

Hereafter, SIZE is defined in units of photons per unit area of the telescope aperture.

Once the geometry of the AS is given, the direction seen by $i$-th pixel is decomposed into two angular components; $\vartheta^{\perp}$ and $\vartheta \|_{i}$ which respectively correspond to the vertical and horizontal axes in Figure 1. The former indicate the angular distance perpendicular to the great circle formed by the SDP, called off-SDP angle. The latter is the component parallel to the AS axis, called off-shower angle. For a known arrival direction of the AS and SDP, DIST parameter represents the angular distance between the arrival direction and observed center of gravity of the all signals. Namely,

$$
(\mathrm{DIST}) \equiv\left\langle\vartheta^{\|}\right\rangle=\sum^{\mathrm{FOV}}\left[\vartheta_{i} \cdot s_{i}\right] /(\mathrm{SIZE})
$$

The second moments for both components; LENGTH and WIDTH are defined as follows:

$$
\begin{aligned}
(\text { LENGTH }) & \equiv \sqrt{\left\langle\vartheta \|^{2}\right\rangle-\langle\vartheta \|\rangle^{2}}=\sqrt{\sum^{\text {FOV }}\left[\left(\vartheta_{i}\right)^{2} \cdot s_{i}\right] /(\text { SIZE })-(\text { DIST })^{2}}, \\
(\text { WIDTH }) & \equiv \sqrt{\left\langle\vartheta^{2}\right\rangle}=\sqrt{\sum^{\text {FOV }\left[\left(\vartheta^{\perp}\right)^{2} \cdot s_{i}\right] /(\text { SIZE })} .}
\end{aligned}
$$

Figure 2 shows examples of the distance dependence of the Cherenkov light images. The colour scale indicates relative signal density. Locations of 20 telescopes are uniformly sampled up to $1.6 \mathrm{~km}$ around the AS axis. The horizontal axes are parallel to $\vartheta \|$ coordinate. Core distance and SIZE are noted. Solid and dashed curves indicate the directions of actual AS maximum and DIST angles along the AS axis, respectively.

In the case of VHECR induced ASs, the angle of Cherenkov light may largely deviate from the AS axis. Thus larger portion of the AS track in wider range of altitudes is observed from the ground level. In addition, Cherenkov light from muons locally landing near IACTs is also detected. With absence of Cherenkov light pool, the maximum core distance that ASs at given energy are detected from is only limited by sensitivities of the detector to SIZE parameter.

However, some Cherenkov images of ASs observed at more than $\sim 1 \mathrm{~km}$ away span up to nearly $30^{\circ}$, requiring ultra-wide-FOV IACT to determine Hillas parameters. They can be used to extract additional information on high energy particle content of the AS and the AS development in general. LENGTH and DIST of AS from VHECRs both increase with increase of core distance without clear cutoff, while WIDTH does not depend much on distance.

For a given AS geometry with respect to the telescope, the DIST angle along AS axis from the arrival direction potentially points towards the direction of the AS maximum. Since the light intensity is in fact nearly proportional to the number of charged particles above the threshold energy $\varepsilon_{\check{C}}$ for Cherenkov emission, SIZE may proportionally reflect such a quantity. Applying the way practiced for the so-called gamma-hadron separation in gamma ray astronomy, the combinations of Hillas parameters can be also used for estimating the chemical composition of VHECRs. 

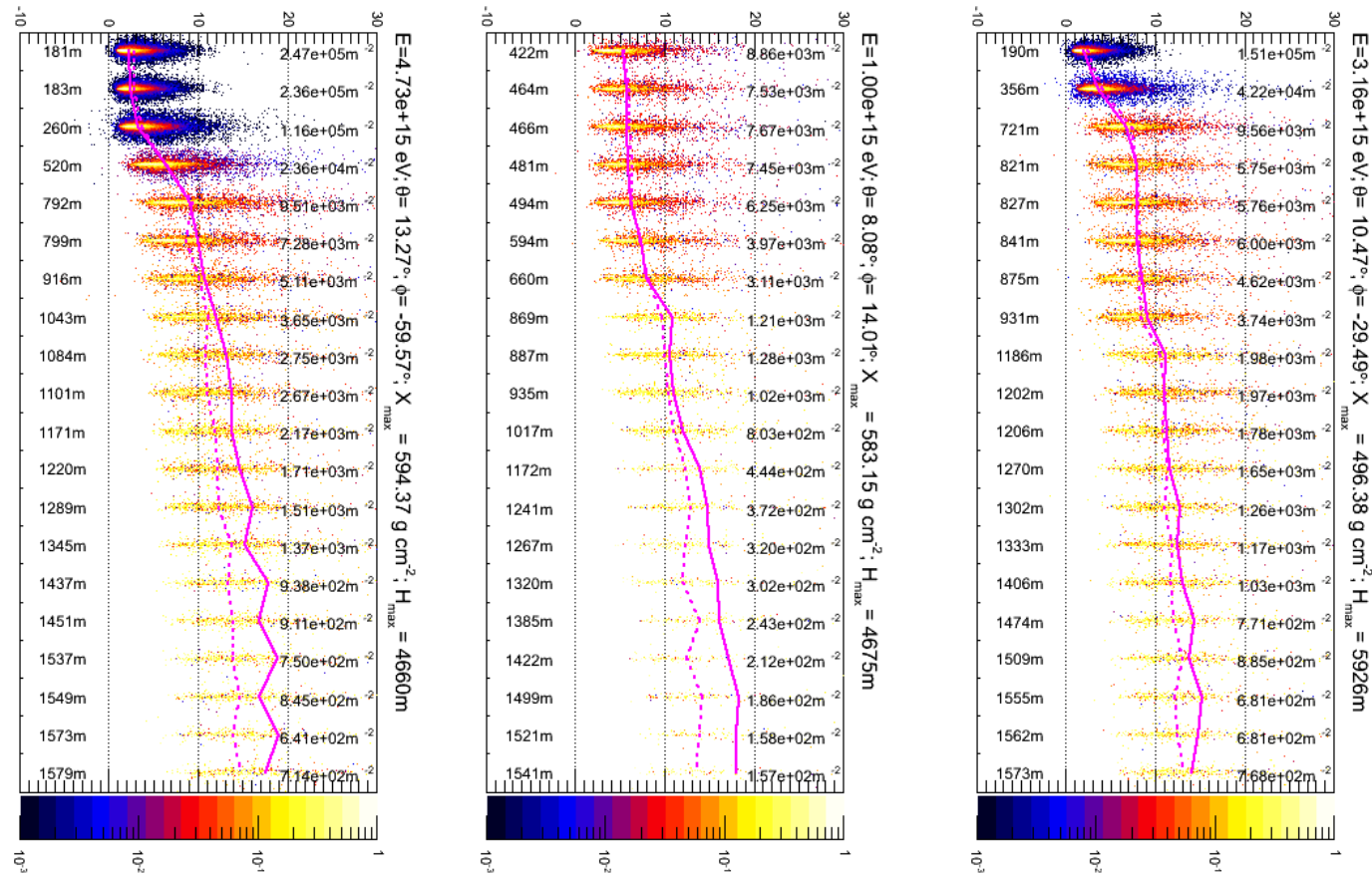

Figure 2: Dependence of Cherenkov image at different distances. Colour scale is relative photon density on the pixel. Core distance and SIZE are indicated. Solid and dashed curves indicate the actual directions of the AS maximum and those pointed the DIST angles, respectively.

Forward Nerling approach In this approach, we aim at reconstructing two-dimensional shower development projected on the plane perpendicular to the SDP. In particular to estimate the type of primary particle, the measurement of $N_{\mathrm{e}}(X)$ is the most relevant that can be example compared with the parametrized Gaissar-Hillas function [16]. Cherenkov images as seen in Figures 1 and 2 are as a result of Cherenkov emission along the AS track and attenuation in the atmosphere. Cherenkov photons are emitted along the track of charged particles with energies exceeding $\varepsilon_{\breve{C}}$. The threshold is a function of refractive index of the atmosphere $n(z)$, where $z$ is the altitude. The Cherenkov yield $Y_{\breve{C}}$, the number of emitted Cherenkov photons per unit column density along the trajectory of an electron with an energy $E_{\mathrm{e}}$, is written as follows:

$$
Y_{\check{\mathrm{C}}}\left(E_{\mathrm{e}}, z\right) \equiv \frac{d n_{\check{\mathrm{C}}}}{d X}=\frac{2 \pi \alpha}{\rho(z)} \int_{\lambda} \frac{\sin ^{2} \theta_{\check{\mathrm{C}}}(z)}{\lambda^{2}} \mathrm{~d} \lambda
$$

where $\alpha$ is the fine structure constant, and $\rho(z)$ is the density of the atmosphere as a function of altitude. Once the energy distribution of electrons in the AS $d N_{\mathrm{e}} / d E_{\mathrm{e}}$ [17] is given, for example the model by Giller et al. [17], the average yield for the AS particles can be estimated.

Figure 3 shows the overview of the Forward Nerling approach. The left panel illustrates the geometry in consideration. Points $\mathrm{O}$ and $\mathrm{A}$ are core and telescope locations, respectively. $\mathrm{C}$ is the emission point of interest. The angle $\vartheta=\angle$ ACO is equal to off-shower angle $\vartheta \|$. On the right, the 

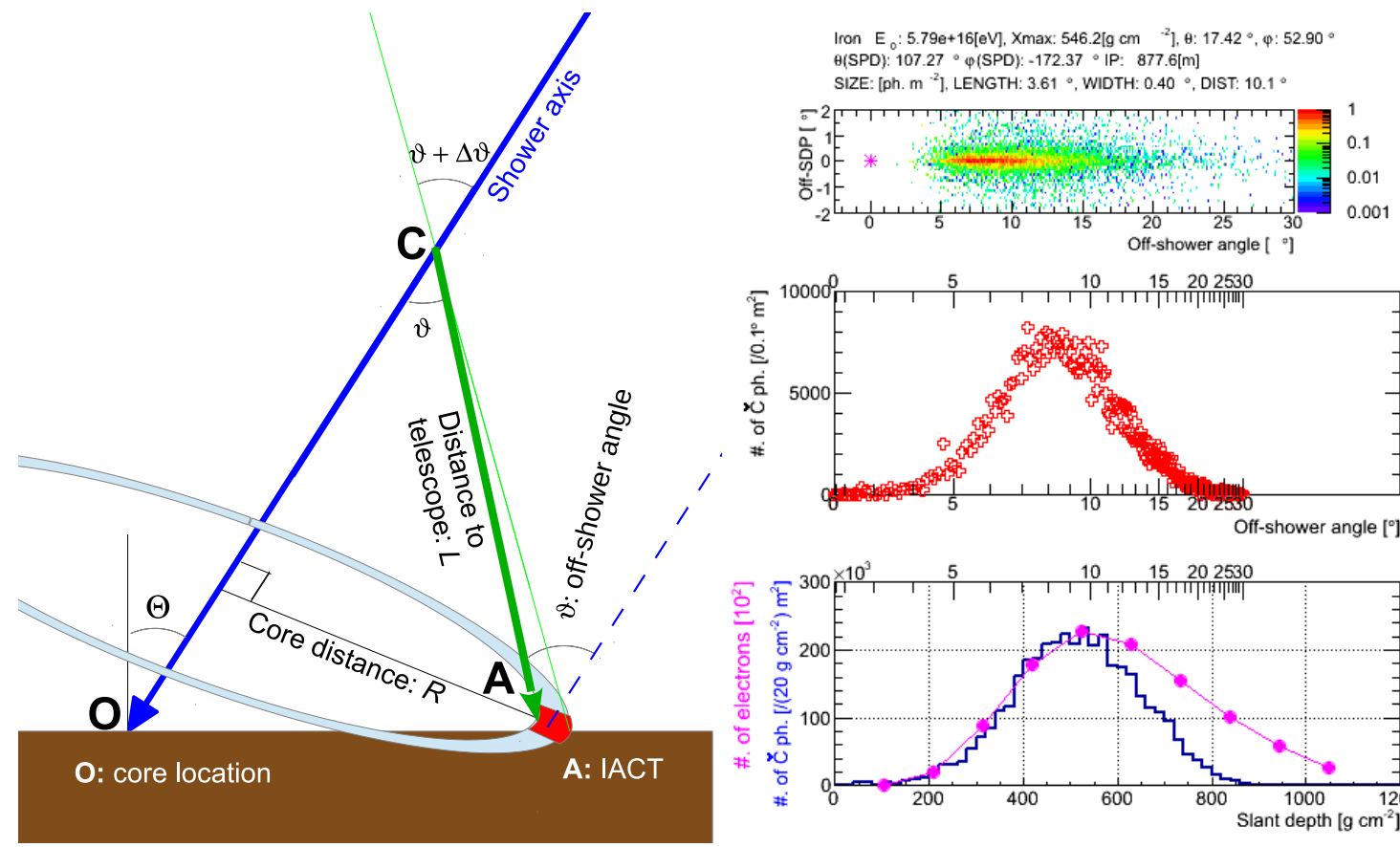

SIZE: [ph. $\mathrm{m}^{-2}$ ]. LENGTH: $3.61^{\circ}$, WIDTH: $0.40^{\circ}$, DIST: $10.1^{\circ}$
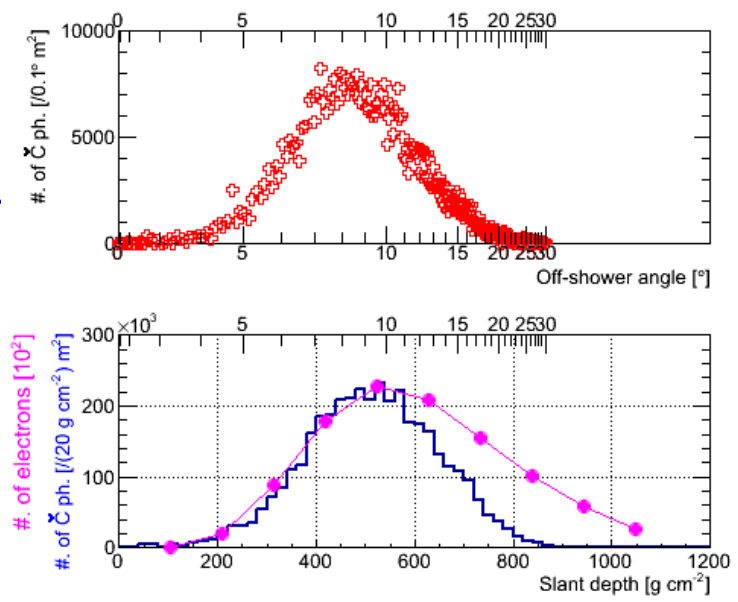

Figure 3: Forward Nerling approach overview. The left panel illustrates the key geometry in consideration. Points $\mathrm{O}$ and $\mathrm{A}$ are core and telescope locations, respectively. $\mathrm{C}$ is the emission point of interest. On the right: top panel is an example of observed Cherenkov image. The middle panel shows the number of emitted photons along the off-shower angle by crosses. The bottom panel shows the number of emitted photons per slant depth along with the number of electrons in the AS by closed circles.

top panel displays an example of observed Cherenkov image. The middle panel shows the number of emitted Cherenkov photons along off-shower angles by crosses. The horizontal axis is scaled to the atmospheric depth along the AS track. The bottom panel shows the number of emitted photons per slant depth along with the number of electrons in the AS by closed circles.

Once the angular distribution of emitted Cherenkov photons $d n_{\check{\mathrm{C}}} / d \vartheta$ with respect to the AS axis are given, the projected profile of the Cherenkov image along off-shower angles can be estimated. Such distribution is formulated by Nerling et al. [18]. For an IACT with radius $D_{\text {tel }}$ at the location A, the estimated number of Cherenkov photons $d s_{\check{C}}$ viewed within off-shower angle $\vartheta$ and $\vartheta+d \vartheta$ is written as follows:

$$
\frac{d s_{\check{\mathrm{C}}}(\vartheta)}{d \vartheta}=\int_{X}\left\{N_{\mathrm{e}}(X) \cdot \int_{\varepsilon_{\check{\mathrm{C}}}}^{\infty}\left[\frac{d N_{e}}{d E_{\mathrm{e}}} \cdot Y\left(E_{\mathrm{e}}, n(z)\right)\right] d E_{\mathrm{e}} \cdot \frac{d n_{\check{\mathrm{C}}}(\vartheta)}{d \vartheta} \cdot \frac{D_{\mathrm{tel}}}{2 \pi R} \cdot \bar{T}\left(z, \vartheta, z_{0}\right)\right\} d X
$$

where $X$ is the atmospheric depth along the AS track and $\bar{T}$ is the transmittance of atmosphere between the reference point $C$ at altitude $z$ and the telescope at $z_{0}$.

Starting from input parameters of $N_{\mathrm{e}}(X)$ function, the number of Cherenkov light emitted per column density along the AS axis $d N_{\check{C}} / d X(X)$, i.e. lines in the same panel, well traces to $N_{\mathrm{e}}(X)$ at the earlier stage of the AS development where the average of $E_{\mathrm{e}}$ is well higher than $\varepsilon_{\check{\mathrm{C}}}$ and then deviates due to decrease of $Y_{\check{\mathrm{C}}}$ according to the lower average $E_{\mathrm{e}}$. Among the Cherenkov photons emitted along the AS track, only a fraction of them direct to the telescope at the point A. Note that $\vartheta$ and $L$ are constrained at each position along the AS track which is determined by the angular 
distribution of Cherenkov photons, e.g. Nerling model, and the ratio of the telescope diameter to the circumstance of circle with a radius $R$. Taking into account the attenuation of light in the atmosphere, the distribution of Cherenkov signals along with the off-shower angle $d s_{\check{\mathrm{C}}}(\vartheta) / d \vartheta$ can be estimated such as seen in the right-middle panel. By searching for input parameters, e.g. $E$ and $A$ of primary CR that fit the observed distribution, the AS event is reconstructed in a form of $N_{\mathrm{e}}(X)$.

\section{Summary and outlook}

We have introduced the basic aspect of VHECR observations using EUSO-type telescopes that have been originally developed for the UHECR observations from space. They have been designed using refractive Fresnel optics to realize ultra wide-FOV in the the framework of the JEM-EUSO mission. Pathfinders telescopes for the mission have been successfully operated. Further studies such as optimization of detector configurations for VHECR physics, in particular study of chemical composition, and experimental activities to test the observational concept are in progress.

Acknowledgment: KS wishes to acknowledge ISDC Data Centre for Astrophysics, University of Geneva for the support of his stay and activities for the present work. We also appreciate computational facilities of ISDC and of RIKEN Integrated Cluster of Clusters (RICC) and Hokusai Great Wave System, operated by RIKEN, Japan.

\section{References}

[1] Apel, W.D. et al. 2013, Astropart. Phys., 47, 54.

[2] Apel, W.D. et al. 2009, Astropart. Phys. 31, 86

[3] Apel, W.D. et al. 2011, Phys. Rev. Lett. 107, 171104.

[4] Amenomori, M. et al. 2008, Astrophys. J., 678, 1165.

[5] Di Scascio, G. 2014, 5th High Altitude workshop, Paris.

[6] Hillas A.M. 2013, Astropart. Phys. 43, 19 (for review).

[7] Picozza, P. al. 2015, in these proceedings, ID0694 and references therein.

[8] von Ballooms, P. et al. 2015, in these proceedings, ID0725 and references therein.

[9] Piotrowski, L.W. et al. 2015, Nucl. Instr. Meth. A, 73, 164.

[10] Halon, W. et al. 2013, Proc. of 33rd ICRC (Rio de Janeiro), ID0964.

[11] Heck, D. et al. 1998, Report FZKA 6019, Forschungszentrum Karlsruhe; http://www-ik.fzk.de/corsika/physics description/corsika phys.html

[12] Bernlöhr, K. 2008, Astropart. Phys. 30, 149

[13] Opstanchenko, S. 2011, Phys. Rev. D83, 014018.

[14] FLUKA, www.fluka.org

[15] Hillas, A.M. 1985, Proc. 19nd ICRC (La Jolla) 3, 445.

[16] Gaisser, T.K. and Hillas A.M. 1977, Proc. 15th ICRC (Plovdiv), 8, 353.

[17] Giller, M. 2005, J. Phys G31, 947.

[18] Nerling, F. et al. 2006, Astropart. Phys., 24, 421. 